\title{
RDUS
}

Revue de DROIT

UNIVERSITÉ DE SHERBROOKE

Titre : $\quad$ APPROCHE COMPARATIVE DU RÈGLEMENT DES CONFLITS ARMÉS AU MOYEN-ORIENT (GAZA ET LIBAN) : À QUELLES CONDITIONS RETROUVER LA STABILITÉ?

Auteur(s): $\quad$ Fady FADEL

Revue : $\quad$ RDUS, 2008-2009, volume 39, numéro 1-2

Pages: $\quad 429-450$

ISSN : $\quad$ 0317-9656

Éditeur: $\quad$ Université de Sherbrooke. Faculté de droit.

URI : $\quad$ http://hdl.handle.net/11143/11535

DOI : $\quad$ https://doi.org/10.17118/11143/11535 
Page vide laissée intentionnellement. 


\section{APPROCHE COMPARATIVE DU RÈGLEMENT DES CONFLITS ARMÉS AU MOYEN-ORIENT (GAZA ET LIBAN) : À QUELLES CONDITIONS RETROUVER LA STABILITÉ?*}

par Fady FADEL**

\section{Introduction}

En moins de deux ans et demi, deux conflits armés sanglants ont éclaté au Moyen-Orient ayant en commun le même belligérant étatique, l'État d'Israël, et deux autres belligérants non étatiques, considérés comme guérillas ou mouvements de résistance, Hezbollah (juillet 2006) et Hamas (décembre 2008).

Or, le nouveau visage que prennent les confrontations entre l'État d'Israël et les nouveaux belligérants est tout autre par rapport aux guerres passées. En effet, d'avril 1950 (la Cisjordanie est annexée par la Transjordanie et l'Égypte prend le contrôle de Gaza) en passant par la deuxième guerre israélo-arabe en octobre 1956 (opération israélienne contre l'Égypte dans le Sinaï suivie d'une intervention franco-britannique à Suez, le 31 octobre pour répondre à la nationalisation du Canal de Suez par Nasser), sans oublier la guerre de juin 1967 (déclenchement de la Guerre des Six Jours). L'armée israélienne occupe le Sinaï, le plateau du Golan, la Bande de Gaza, la Cisjordanie et Jérusalem-est) et celle d'octobre 1973 (Guerre d'octobre [Guerre du Kippour] déclenchée par les Égyptiens et les Syriens pour reconquérir les territoires occupés par Israël. Victoire d'Israël qui, un instant menacé, a repris l'initiative au bout d'une semaine de combats), on avait affaire à des entités étatiques en conflits directs. Vu le rapport de force et l'appui important américain à l'État d'Israël, c'est ce dernier qui avait le dernier mot dans ces confrontations guerrières. Sauf que, depuis

*. $\quad$ Conférence donnée à l'Université de Sherbrooke, le 18 février 2009.

** . Professeur à l'Université Antonine-UPA, Beyrouth, Liban. 
le début des années 80 , on a changé de stratégie de combat du côté arabe - et il semble qu'on y reviendrait prochainement et éventuellement, mais autrement (dernier sommet des pays arabes au Koweït en janvier 20091) - et ce sont désormais les factions militaires "non gouvernementales", dites les forces de résistance contre l'occupant, qui prennent en charge le combat militaire contre l'État d'Israël. Deux exemples illustrent cette nouvelle stratégie "tactique»: la guerre de juillet 2006 entre Israël et Hezbollah et la guerre de décembre 2008 entre Israël et Hamas.

Mais, contrairement aux apparences, il s'agit à nos yeux de deux conflits différents, tant dans la raison profonde de leur éclatement (première partie) que dans les termes de leur résolution (deuxième partie). En effet, bien que la diplomatie multilatérale se soit mobilisée avec force pour trouver une issue compromissoire à ces guerres (cessation des hostilités et cessez-lefeu), il n'en demeure pas moins qu'il existe des différences de fond et de forme dans le règlement adopté dans chacune des deux guerres.

Cette approche comparative tâchera de révéler les enjeux de la diplomatie multilatérale quant au rôle d'apaisement joué dans les cas de Gaza et du Liban, tout en soulignant les divergences concernant les résultats escomptés quand il s'agit d'une diplomatie onusienne inscrite dans la durée face à une diplomatie extra-onusienne, tributaire de tractations interétatiques.

\section{1- Ressemblances et différences entre les guerres de 2006 et de 2008 : les raisons des conflits}

L'histoire des conflits armés d'Israël avec Hezbollah et Hamas n'est pas nouvelle. Durant l'occupation israélienne du territoire libanais jusqu'à l'année 2000, les opérations militaires, de part et d'autre, ne manquaient pas entre Israël et Hezbollah. La

1. Communiqué final du Sommet des Rois et chefs d'État arabes et commentaire dans le journal An-Nahar, mercredi 21 janvier 2009, chronique d'Émile Khoury, page 3. 
dernière en date est celle des "raisins de colère" en 1996. Il en est de même dans le cas de Hamas en 2006. Quoique, les derniers conflits armés de 2006 et de 2008 sont caractéristiques au niveau du contexte interne et régional, tant au Liban (a) qu'à Gaza (b). Ceci n'est pas sans conséquence quant aux divergences profondes entre ces deux cas (c).

\section{a- Le cas du Liban}

Après le retrait israélien d'une grande partie du Liban-sud en 2000 et le tracé de la Ligne bleue (délimitant provisoirement la frontière libano-israélienne), il fut indiqué expressément que l'armée israélienne occupe toujours une partie du territoire de l'État libanais au nord du village Ghajar, en vertu des frontières libano-israéliennes reconnues internationalement ${ }^{2}$. Quant aux fermes de Chébaa, occupées en 1967, lors de l'occupation et de l'annexion par Israël du Plateau du Golan syrien, il s'avère que ce territoire "arabe" occupé fait partie formellement, selon les cartes déposées au siège de l'ONU à New York, du territoire syrien. Autrement dit, au vu des documents internationaux pertinents, les fermes de Chébaa se trouvent, par erreur, à l'intérieur du territoire syrien et de ses frontières internationalement reconnues.

Dans la résolution 1701/2006, le secrétaire général des Nations Unies est invité à présenter un examen approfondi relatif à cette question territoriale ${ }^{3}$. Les rapports qui s'en suivront décrivent les étapes parcourues jusqu'à présent par le Secrétaire général et son équipe de cartographes. ${ }^{4}$

En outre, force est de reconnaître qu'il y avait des prisonniers "politiques" libanais en Israël qui purgeaient des

2. Résolution concernant la situation au Moyen-Orient, Doc. off. CS NU, 61e année, $5511^{\mathrm{e}}$ séance, Doc. NU S/RES/1701 (2006) au par. 5 [Résolution $1701]$.

3. Ibid. au par. 10.

4. Rapport du Secrétaire général sur l'application de la résolution 1701 (2006) du Conseil de sécurité, Doc. off. CS NU, 2008, Doc. NU S/2008/715, au par. 60 [Rapport du Secrétaire général]. 
peines de prison à la suite d'opérations de libération et de résistance à l'occupant israélien.

Dans ce contexte intervient l'opération du Hezbollah en juillet 2006 qui consiste à enlever deux soldats israéliens au-delà de la Ligne bleue, à l'intérieur du territoire israélien, en vue de provoquer un échange par la suite avec des prisonniers libanais en Israël, ce qui a appelé d'ailleurs à une condamnation par le Conseil de sécurité de l'ONU55.

La riposte israélienne fut d'envergure et disproportionnée, au nom de la légitime défense, pour la libération des soldats israéliens enlevés et surtout pour mettre fin à l'existence du Hezbollah (1300 morts côté libanais et 33 morts côté israélien, sans parler des blessés et des dégâts structurels et infrastructurels au Liban). Ces objectifs furent profondément critiqués par la commission d'enquête gouvernementale israélienne Winograd qui a considéré que "certains des buts déclarés de la guerre n'étaient pas clairs et ne pouvaient pas être atteints" et "la décision de répondre (à la capture de deux soldats israéliens) par une frappe militaire immédiate et intense ne se fondait pas sur un plan militaire détaillé, complet et autorisé»6.

À cet effet, il convient de signaler que le Hezbollah était représenté dans le gouvernement libanais à travers le ministre de l'Énergie, M. Mohammad Fneych, ainsi qu'à travers ses alliés

5. Organisation des Nations Unies, Compte rendu CS/8776, "Conseil de sécurité : La majorité des membres reconnaît la responsabilité du Hezbollah dans la crise au Liban tout en condamnant le caractère disproportionné de la riposte israélienne" (14 juillet 2006), en ligne : <http://www.un.org/News/fr-press/docs/2006/CS8776.doc.htm>.

Toutes références tirées de l'Internet ont été consultées et vérifiées le 30 mars 2009.

6. http://contreinfo.info/article.php3?id_article=1023 ou http://www. humanite.fr/-+2007-05-02_International+-.

Pour le rapport intégral, voir : http://www.mfa.gov.il/MFA/MFAArchive/ 2000_2009/2008/Winograd\%20Committee\%20submits\%20final\%20repo rt\%2030-Jan-2008. 
traditionnels, le mouvement Amal, dont le président de la Chambre des députés, Nabih Berri, est le chef.

Cette dernière mention relative à la présence du Hezbollah dans le gouvernement constitue l'un des points fondamentaux de différence avec le Hamas lors du conflit armé l'opposant à Israël.

\section{b- Le cas de Gaza}

"Il est clair qu'Israël n'acceptera qu'un règlement qui prévoie une cessation complète des tirs de roquettes, la fin de réarmement du Hamas et une vie normale pour les citoyens du Sud d'Israël. Nous ne parviendrons à un apaisement que si toutes les exigences d'Israël sont satisfaites", a déclaré le dimanche 4 janvier 2009 Ovid Yehezqel, secrétaire général du gouvernement israélien. ${ }^{7}$

La première roquette a été tirée au début de l'année 2001, quelques mois après le début de la deuxième Intifada, le 29 septembre 2000. Au début des tirs, leur nombre est resté relativement limité à l'exception de 2004, où 281 roquettes avaient été tirées ainsi que 250 obus de mortiers. Un premier pic a été atteint en 2006 avec 946 tirs, avant une légère décrue en 2007 puis en 2008.

En se retirant de la bande de Gaza en 2005, et en démantelant les colonies juives qui y étaient implantées, Ariel Sharon pensait en finir avec les opérations incessantes dans ce territoire, et soulager l'armée de ce fardeau.

Moins de cinq ans plus tard, ses successeurs doivent faire face à une nouvelle menace à Gaza. Le retrait unilatéral de Sharon, sans négociations préalables avec l'Autorité palestinienne, avait été présenté comme une victoire par le Hamas. Le

7. Michel Bôle-Richard, "Un conflit provoqué par les tirs de Qassam sur Israël et par le blocus israélien de Gaza", Le Monde (6 janvier 2009) à la p. 6. 
mouvement islamiste avait remporté l'année suivante les élections législatives palestiniennes, le 25 janvier 2005, avant de prendre le pouvoir par un coup de force à l'été 2007, évinçant de Gaza les forces du président de l'Autorité palestinienne, Mahmoud Abbas.

À cet effet, il convient de signaler que cette Autorité palestinienne, composée de président, de gouvernement et de Parlement, a été créée en vertu des accords d'Oslo en 1993 qui ont donné un embryon d'autonomie aux territoires palestiniens. Il s'agit de la seule instance politique représentant tous les Palestiniens et reconnue par la communauté internationale ${ }^{8}$. Par conséquent, l'on se trouve désormais devant deux gouvernements: le gouvernement de Hamas à Gaza, qui a remporté les dernières élections législatives, et le gouvernement de Fateh en Cisjordanie, constitué de fait à la suite du coup de force de Hamas en 2007.

Dans un premier temps, Israël avait réussi à circonscrire la menace représentée par les kamikazes, dont le Hamas avait fait sa spécialité, par une clôture électrifiée et étroitement surveillée entourant la bande de Gaza. Dans ce contexte de confrontations, Israël avait organisé un étroit blocus terrestre et maritime du territoire palestinien, destiné à faire plier le Hamas.

Ce système a été contourné par les militants islamistes sous terre et dans les airs. En creusant des tunnels, le Hamas avait lancé des attaques contre les postes israéliens, comme celle qui avait abouti à la capture du caporal Gilad Shalit en juin 2006, depuis lors toujours prisonnier à Gaza. Ces souterrains ont aussi permis de faire entrer, depuis un Sinaï égyptien mal contrôlé, armes et ravitaillement, et notamment des roquettes plus sophistiquées que les engins artisanaux bricolés dans les ateliers de chaudronnerie du Hamas. Dans les airs ensuite, Hamas faisait pleuvoir par-dessus la barrière terrestre des projectiles à la portée de plus en plus longue contre les villes du Sud d'Israël.

8. Doc. off. AG NU, 1997, 68e séance plén., Doc. NU A:52:L.52 et Corr. et Add. 1. 
Pour le mouvement islamiste, les tirs de roquettes ne sont que des ripostes au blocus infligé par Israël contre son territoire, qui dépend étroitement des marchandises transitant par son voisin et dont la population vit sous perfusion d'aide internationale. Quoique relativement inefficaces, ces tirs sont pour Israël une menace inacceptable.

L'armée israélienne a fini par se résoudre à retourner dans Gaza, où elle n'avait plus pénétré depuis la décevante opération "Pluie d'été" de 2006, qui avait échoué à libérer le caporal Shalit. L'objectif officiel de l'opération "Plomb durci" n'est pas aujourd'hui de réoccuper Gaza et d'administrer 1,5 million de Palestiniens, mais de mettre fin aux tirs du Hamas contre Israël. Le Hamas s'est préparé depuis l'été 2007 à une confrontation de ce genre. Le mouvement, qui met le sacrifice individuel, volontaire ou non, au centre de son identité, représente un adversaire plus coriace que les militants du Fateh palestinien qu'Israël avait réduits dans les opérations en Cisjordanie pendant la seconde Intifada en 2002. Le Hamas a augmenté son arsenal, creusé des caches et des tunnels, dissimulé des mines et préparé ses combattants. L'offensive terrestre devait, selon l'état-major israélien ${ }^{9}$, porter un coup définitif aux capacités militaires du Hamas. Elle a commencé après une semaine d'opérations aériennes, où viennent une nouvelle fois d'être démontrées les limites de l'aviation face à un adversaire furtif. Malgré leur précision, les bombes guidées et les missiles se sont révélés incapables de faire cesser totalement les tirs de roquettes et de mortiers. Les drones, capables de filmer de nuit ou sous un couvert nuageux à la caméra thermique les moindres mouvements de l'adversaire, ne parviennent pas à tout savoir d'un mouvement maladivement secret et clandestin.

9. Adrien Jaulmes, "Pourquoi Gaza a replongé dans la guerre», Le Figaro (5 janvier 2009), en ligne : Le Figaro.fr <http ://www.lefigaro.fr/international/2009/01/05/01003-0090105ART FIG00247-pourquoi-gaza-a-replonge-dans-la-guerre-.php>. 


\section{c- Première synthèse}

- Bien que le Hamas et le Hezbollah jouissent tous les deux de légitimité représentative démocratique, il n'en demeure pas moins qu'au moment du conflit armé l'on assiste à un démembrement de l'exécutif $\mathrm{du}$ côté palestinien (deux gouvernements sur deux territoires "séparés") alors qu'il s'agit d'un seul gouvernement solidaire intégrant le Hezbollah du côté libanais.

- Malgré les condamnations ayant émané de tous bords à l'encontre de l'action "illégale» du Hezbollah dans la capture des soldats israéliens le 12 juillet 2006, force est de constater, à l'instar du secrétaire général des Nations Unies, que la question de libération des prisonniers libanais en Israël et des soldats israéliens enlevés est totalement réglée. Autrement dit, "après 18 mois d'efforts intensifs (diplomatiques), les aspects humanitaires de la résolution 1701/2006 avaient été remplies» ${ }^{10}$.

En outre, il convient de rappeler qu'à l'époque, le Hezbollah, qui est sorti victorieux des élections législatives en 2005 faisant alliance avec la coalition du Courant du Futur (présidé par Saad Hariri) et du Parti Progressiste Socialiste (présidé par Walid Joumblatt), jouissait d'une certaine couverture nationale quant à ses mesures de résistance et de libération des prisonniers libanais en Israël.

Du côté du Hamas, les efforts diplomatiques restent timides au sujet des prisonniers palestiniens en Israël ou en ce qui concerne le soldat israélien enlevé Gilad Shalit. De même, l'on est loin de considérer que les actions militaires de Hamas reçoivent l'aval

10. Rapport du Secrétaire général, supra note 5 au par. 57. 
(2008-09) 39 R.D.U.S. Approche comparative du règlement

au Moyen-Orient (Gaza et Liban)

de la classe politique et de l'ensemble de la population palestinienne (deux factions palestiniennes séparées sur deux territoires juxtaposés, Gaza et Cisjordanie), comme l'a souligné le 1er février 2009 en Égypte le président de l'Autorité palestinienne, Mahmoud Abbas ${ }^{11}$.

- Bien que la Syrie et l'Iran prétendent constituer les puissances régionales "d'objection" à l'encontre d'Israël et appuient avec force les guérillas locales ainsi que les mouvements de résistance (Hamas et Hezbollah), il n'en reste pas moins que les conséquences des actions militaires de ces deux belligérants non étatiques sont différentes et ils (Hamas et Hezbollah) doivent prendre en compte les facteurs sociologiques relatifs à la composition spécifique de chacune des sociétés à laquelle ils appartiennent (la Palestine étant "uniculturelle»démocratie majoritaire; le Liban étant multicultureldémocratie consociative).

- Bien que Hezbollah et Hamas constituent les deux belligérants directs face à Israël, Hezbollah ne s'est pas constitué formellement en partie principale lors des négociations qui ont amené l'adoption de la résolution 1701. C'est le gouvernement libanais qui assurait la représentativité du Liban sur la scène diplomatique internationale, que ce soit à l'ONU ou ailleurs, et qui a négocié et accepté le règlement tel que mentionné expressément dans la résolution. En revanche, Hamas a négocié lui-même et directement, en tant que mouvement de résistance et

11. "Pour Abbas, le Hamas a "risqué la vie du peuple" palestinien", Le Monde avec AFP (1 er février 2009), en ligne : Le Monde.fr $<$ http ://www.lemonde.fr/cgi-bin/ACHATS/acheter.cgi?offre=ARCHIVES \&type_item=ART_ARCH_30J\&objet_id=1068748\&clef=ARC-TRK-NC_01\#e ns_id=1106055>. 
"gouvernement", les termes du règlement, et ce, à travers le rôle de médiation assuré par l'Égypte.

Où en sommes-nous aujourd'hui?

\section{2- Quels règlements adéquats des conflits?}

En stratégie militaire, les conflits armés prennent fin à la suite d'un règlement permettant au plus fort d'imposer son approche, ou au moins de se mettre à la table de négociation muni de positions de puissance se référant au rapport de force confirmé sur le terrain. Or, dans les deux guerres de 2006 et de 2008-2009, les règlements sont le fruit d'une action diplomatique ayant abouti - jusqu'à présent - à une cessation des hostilités. Dans ce sillage, il nous semble que dans le cas libanais, les efforts diplomatiques ont abouti à l'adoption par le Conseil de sécurité de la résolution 1701/2006 (a), alors que dans le cas de Gaza, c'est la résolution 1860/2009 qui devrait servir, voire aboutir à une (plusieurs) action(s) diplomatique(s) multilatérale(s) (b). Quelles en sont alors les conséquences? (c)

\section{a- Principaux apports de la résolution 1701/2006}

- L'action diplomatique multilatérale qui a rassemblé la Ligue des États arabes, l'Union européenne, les cinq États membres permanents du Conseil de sécurité s'est conjuguée avec les efforts nationaux du gouvernement libanais et du commandement en chef de l'armée libanaise. En effet, ce n'est qu'à la suite du plan en sept points annoncé par le président du Conseil des ministres libanais Fouad Siniora le 27 juillet 2006 à la conférence de Rome en Italie sur le Liban et adopté unanimement par le gouvernement libanais ${ }^{12}$, notant pour la première

12. Adrien Jaulmes, "Beyrouth réclame un retrait israélien du LibanSud", Le Figaro (15 octobre 2007), en ligne : Le Figaro.fr 
fois la volonté d'étendre l'autorité du gouvernement sur son territoire par l'intermédiaire de ses propres forces armées légitimes ${ }^{13}$, qu'a eu lieu l'adoption par le C.S. de la résolution 1701/2006 qui a mis fin aux hostilités.

- Le fait que le Conseil de sécurité ait qualifié la situation au Liban comme étant "une menace à la paix et à la sécurité internationales»14 donne à sa résolution une ampleur décisionnelle qui s'impose aux parties au conflit, sans qu'il y ait une mention expresse du chapitre VII de la Charte. Autrement dit, les paragraphes 7 , où l'on lit que le C.S "Affirme", 11, où il est dit que le C.S. "décide", 12, où le C.S. "autorise", 15 et 16, où le C.S. "décide toujours", jouissent d'une autorité s'imposant aux belligérants et à l'ensemble des États puisqu'il s'agit d'une action

$<$ http ://www.lefigaro.fr/international/2006/08/07/01003-20060807AR TFIG90126-beyrouth_reclame_un_retrait_israelien_du_liban_sud.php $>$. Le plan en sept points : 1- un engagement à relâcher les prisonniers libanais et israéliens par le canal du Comité international de la CroixRouge. 2- le retrait de l'armée israélienne derrière la ligne "bleue" (tracée par l'ONU entre le Liban et Israël) et le retour des déplacés dans leurs villages. 3- un engagement du Conseil de sécurité à placer le secteur des fermes de Chébaa sous juridiction des Nations Unies. 4- le déploiement de l'autorité du gouvernement libanais sur son territoire au moyen de ses propres forces armées légitimes. 5- le renforcement des forces internationales des Nations Unies opérant dans le sud du Liban en nombre, équipements, mandat et périmètre d'opérations autant que nécessaire pour entreprendre le travail humanitaire urgent et les opérations de secours. 6- l'engagement des Nations Unies à mettre en œuvre l'accord d'armistice signé par le Liban et Israël en 1949. 7- la communauté internationale s'engage à soutenir le Liban à tous les niveaux et à l'aider à supporter l'immense fardeau résultant de la tragédie humaine, sociale et économique qui l'a frappé. Pour le plan en sept points, voir le quatrième rapport semestriel du secrétaire général de l'ONU sur l'application de la résolution 1559/2004, S/2006/832 (paragraphes : 18, 19, 25, 49) : http://www.unicbeirut.org/ unic_editor/Download.asp?table_name=uploadDocs\&field_name=id\&FileI $\mathrm{D}=6$. Voir aussi : http://www.voltairenet.org/article142811.html.

13. Résolution 1701, supra note 3, préambule.

14. Ibid. 
s'inscrivant dans le cadre d'une "menace contre la paix et la sécurité internationales", et d'une "décision"15, au sens de l'article 25 de la Charte"16. Faut-il rappeler par exemple que ce concept est bel et bien l'intitulé du chapitre VII de la Charte, où le C.S envisage des mesures coercitives s'imposant à tous.

- La résolution 1701 renforce le mandat de la Force intérimaire des Nations Unies au Liban (FINUL 2) qui dispose désormais d'une mission d'assistance vis-àvis du gouvernement libanais pour "veiller à ce que son théâtre d'opérations (son lieu de déploiement du sud du fleuve de Litani jusqu'à la Ligne bleue) ne soit pas utilisé pour des activités hostiles de quelque nature que ce soit, à résister aux tentatives visant à l'empêcher par la force de s'acquitter de ses obligations dans le cadre du mandat que lui a confié le C.S. [...]". En outre, il y est fait mention que la FINUL doit "aider les forces armées libanaises (FAL) à prendre les mesures en vue de l'établissement de la zone d'exclusion de tous personnels armés, biens et armes autres que ceux déployés dans la zone par le Gouvernement libanais et les forces de la FINUL"17.

Il fut procédé à l'élargissement du mandat de la FINUL, en vertu des règles d'engagement adoptées aux Nations Unies par l'ensemble des États participants à la FINUL et relatives à l'emploi de la

15. Nguyen Quoc Dinh, Patrick Daillier et Alain Pellet, Droit international public, 7e éd., Paris, L.G.D.J., 2002 à la p. 847. Voir également Conséquences juridiques pour les États de la présence continue de l'Afrique du Sud en Namibie (Sud-Ouest africain) nonobstant la résolution 276 (1970) du Conseil de sécurité, Avis consultatif, [1971] C.I.J. rec. 16 à la p. 53.

16. Charte des Nations Unies, 26 juin 1945, R.T. Can. 1945 no 7, art. 25 : "Les Membres de l'Organisation conviennent d'accepter et d'appliquer les décisions du Conseil de sécurité conformément à la présente Charte."

17. Résolution 1701, supra note 3 aux par. 8, 11 et 12 . 
force et à la liberté totale de circulation dans sa zone de déploiement. 18

- $\quad$ La résolution 1701 consacre désormais et au premier rang le rôle joué par l'action diplomatique sous l'égide de l'ONU, par le biais du Secrétariat général, en vue du règlement pacifique des questions relatives à l'établissement d'un cessez-le-feu durable, aux prisonniers libanais en Israël, aux soldats israéliens enlevés, aux fermes de Chébaa, à la partie libanaise village de Ghajar, à la sécurisation des frontières libanaises, etc.

\section{b- Les apports de la résolution 1860/2009 "en question"}

- $\quad$ Il convient de signaler en premier lieu que le C.S., usant de son pouvoir discrétionnaire de qualification, en vertu de la Charte de l'ONU à l'article 39, n'a pas procédé à qualifier la situation à Gaza comme étant une menace contre la paix et la sécurité internationale (comme il le fait fréquemment dans le cas libanais). Cela aurait donné davantage d'envergure, ne serait-ce que théoriquement, à la résolution en question.

S'inscrivant dans un cadre de médiation - non contraignant - en appelant les parties au conflit de résoudre pacifiquement leur différend, le C.S. recommande, à travers la résolution 1860, sans pouvoir de décision, les termes de la fin du conflit. En effet, en utilisant à plusieurs reprises la formule "le C.S. souligne, appelle (trois fois), condamne, encourage, demande", force est de constater que nous sommes en face d'une résolution à "caractère exhortatif" bien différente "des décisions à caractère

18. Documents UN Restricted et UN Confidential. Fady Fadel, "De la FINUL I à la FINUL II : un nouveau mandat "robuste"» dans Jocelyn Coulon, dir., Guide du maintien de la paix 2009, Montréal, Athéna, 2009, p. 29. 
d'injonction" 19 , dont il est question à l'article 25 de la Charte.

Autrement dit, l'opposabilité juridique de la résolution en question quant à ses dispositifs concernant les belligérants est en cause. Le seul paragraphe où le C.S. "décide», et que les Etats membres conviennent d'accepter et d'appliquer, concerne le fait "de rester saisi de la question". Du reste, il s'agit de recommandations clairement formulées par le C.S. qui nécessiteraient leur acceptation par les parties en vue de leur application. Autrement dit, cette résolution "d'inspiration conciliatrice" supposait "le consensus ou au minimum l'acceptation des États concernés»20. Israël ne l'a pas fait, en refusant d'appliquer d'entrée de jeu la résolution $1860^{21}$ et en demandant auparavant aux États-Unis de s'abstenir lors du vote de la résolution en question ${ }^{22}$.

Ainsi, nous remarquons que le cessez-le-feu auquel a appelé le C.S. dans sa résolution au paragraphe 1 en date du 8 janvier 2009 n'a donné aucun effet dans

19. Erik Suy, "Commentaire de l'article 25 de la Charte" dans Jean-Pierre Cot et Alain Pellet, dir., La Charte des Nations Unies : Commentaire, article par article, Paris, Economica, 1991 à la p. 476.

20. Jean Combacau et Serge Sur, Droit international public, 6e éd., Paris, Montchrestien, 2004 à la p. 636.

21. "Israël refuse un cessez-le-feu" (13 janvier 2009), en ligne : Topnet.tn <http://www.topnet.tn/index.php?option=com_content\&task=view\&id=8 4\&Itemid=377\&alDate $=2009-01-13 \&$ type $=$ actualite-internationale \&ligne Article $=17>$. Voir aussi Agence de presse Xinhua, "La Chine s'inquiète sérieusement de la non application de la Résolution 1860 de l'ONU sur Gaza" (14 janvier 2009), en ligne: French.China.org.cn $<$ http ://french.china.org.cn/conferencedepresse/2009-01/14/content_ 17103622.htm>.

22. Hilary Leila Krieger, "Résolution 1860 : Rice nie les déclarations d'Olmert" Jerusalem Post (15 janvier 2009), en ligne : Jerusalem Post.com <http ://fr.jpost.com/servlet/Satellite?pagename=JFrench/ JPArticle/ShowFull\&cid=1231950857763>. 
l'immédiat. Bien au contraire, ce n'est que le 18 janvier 2009, à travers une décision unilatérale gouvernementale israélienne, qu'un cessez-le-feu fut adopté par l'État d'Israël, suivi quelques heures après par une décision unilatérale de Hamas de décréter un cessez-le-feu, sans aucune référence à la résolution $1860^{23}$.

Contrairement à la résolution 1701, la 1860 ne prévoit pas de mécanisme de contrôle du cessez-lefeu auquel elle appelle les parties. Bien au contraire, la 1860 encourage la diplomatie extra-onusienne à prévoir les termes du règlement et les mécanismes appropriés pour le cessez-le feu ${ }^{24}$, et opère du coup une délégation "implicite» de compétences à l'endroit d'États et non pas d'organisations régionales. En effet, si le C.S. avait qualifié la situation à Gaza comme étant une menace contre la paix et la sécurité internationales (se fondant par exemple sur la qualification des attaques comme étant terroristes à l'encontre d'Israël en provenance de Hamas à Gaza, cf. les résolutions 1269 en 1999, 1368, 1373 et 1377 en 2001), sa délégation de compétences aurait été explicite et constitué un précédent.

Par conséquent, l'ancienne secrétaire d'État américaine Condoleezza Rice et la ministre des Affaires étrangères israélienne Tzipi Livni ont paraphé le vendredi 16 janvier 2009 un accord bilatéral destiné à favoriser la lutte contre la contrebande d'armes vers la bande de Gaza, dans le

23. "Après Israël, le Hamas annonce un cessez-le-feu à Gaza", La Tribune (18 janvier 2009), en ligne: La Tribune.fr <http://www.latribune.fr/actualites/economie/international/20090118t rib000333004/apres-israel-le-hamas-annonce-un-cessez-le-feu-a-gaza. html>.

24. Doc. off. CS NU, 64e année, 6063e séance, Doc. NU S/RES/1860 (2009) aux par. 6-8. 
cadre des efforts pour parvenir à un cessez-le-feu. ${ }^{25}$ De leur côté, l'Allemagne, la France et le RoyaumeUni se sont dits prêts à contribuer à la lutte contre la contrebande d'armes vers la bande de Gaza dans une lettre commune envoyée aux dirigeants israélien et égyptien et rendue publique samedi par le gouvernement allemand à Berlin. "Nous avons dit notre disposition à prendre une série de mesures qui devraient mener à la fin de la contrebande d'armes vers Gaza", ont écrit la chancelière allemande Angela Merkel, le président français Nicolas Sarkozy et le premier ministre britannique Gordon Brown dans cette lettre adressée au premier ministre israélien Ehud Olmert et au président égyptien Hosni Moubarak. "Nous nous sommes étroitement concertés ces derniers jours et allons coopérer avec les gouvernements israélien et égyptien pour appliquer ces mesures", ajoutent-ils dans la lettre transmise le samedi 17 janvier 2009 à ses destinataires par les ambassades à Jérusalem et au Caire. ${ }^{26}$ Le lendemain, le dimanche 18 janvier 2009, le sommet de Charm el-Cheikh a lieu dans le cadre $\mathrm{du}$ renforcement du cessez-le-feu, sans oublier les déclarations du nouvel émissaire américain au Moyen-Orient Georges Mitchell concernant la consolidation de la trêve à Gaza ${ }^{27}$. En un mot, c'est la diplomatie bilatérale et multilatérale extraonusienne qui intervient au premier chef dans le

25. "Rice et Livni signent un accord contre la contrebande d'armes à Gaza", NouvelObs (17 janvier 2009), en ligne: NouvelObs.com <http://tempsreel.nouvelobs.com/speciales/international/le_conflit_a_g aza/20090116.OBS9955/rice_et_livni_signent_un_accord_contre_la_cont rebande_d.html>.

26. Voir : http://www.rtlinfo.be/rtl/archive/article/211368/?\&archiveYear= 2009.

27. "George Mitchell veut "consolider" la trêve à Gaza : Chronologie», Le Monde (30 janvier 2009), en ligne: Le Monde.fr <http:// www.lemonde.fr/web/recherche_breve/1,13-0,371067974,0.html>. 
règlement $\mathrm{du}$ conflit israélo-gazaouite, se servant notamment des documents internationaux pertinents à cet effet.

Enfin, contrairement à la résolution 1701, d'une part, nous constatons une absence totale de mention dans la résolution 1860 concernant la remise de rapports périodiques du secrétaire général des Nations Unies, relatifs à l'application de la résolution en question; cela montre encore une fois que l'application de ladite résolution ne jouit pas d'un cadre international contraignant. D'autre part, il n'y est fait aucunement référence à la libération du soldat israélien enlevé Gilad Shalit et aux prisonniers palestiniens en Israël, bien qu'aux paragraphes 3 et 4 de la 1860, le C.S. s'intéresse de près à la question humanitaire.

\section{c- Deuxième synthèse}

- Le déploiement d'une force internationale de maintien de la paix au Liban-Sud, chargée d'assister les forces armées libanaises dans la garantie de la stabilité et de la cessation des hostilités entre Israël et le Liban, constitue l'une des grandes différences avec les efforts diplomatiques extra-onusiens visant la consolidation d'un cessez-le-feu à Gaza, déjà précaire dès le départ, puisqu'il est déclaré unilatéralement par les parties au conflit.

En effet, le secrétaire général des Nations Unies souligne dans son rapport sur l'application de la résolution 1701 le 18 novembre 2008 que "la cessation des hostilités entre Israël et le Liban est toujours respectée et dans l'ensemble, la situation militaire, de même que la situation relative à la sécurité dans la zone d'opérations de la Force 
intérimaire des Nations Unies au Liban (FINUL) est restée calme. Les parties respectent toujours la Ligne bleue d'une façon générale» 28 .

À la question de savoir si Israël a atteint les objectifs de son opération militaire, des analystes et des politologues considèrent que malgré le fait que Hamas a été affaibli dans cette guerre, il n'en demeure pas moins qu'il a toujours la capacité et la possibilité de lancer des roquettes et des obus vers Israël29. Bien plus, les mécanismes de contrôler les contrebandes d'armes ou de permettre à Gaza d'être une zone exclue d'armes et de personnes armées,

28. Rapport du Secrétaire général, supra note 5 au par. 6 .

29. Une synthèse de ces points de vue se trouve sur les liens suivants :

Elise Barthet, "Après l'offensive sur Gaza, Israël s'interroge sur sa stratégie", Le Monde (19 janvier 2009), en ligne: Le Monde.fr $<$ http ://www.lemonde.fr/cgi-bin/ACHATS/acheter.cgi?offre=ARCHIVES \&type_item=ART_ARCH_30J\&objet_id=1066999\&clef=ARC-TRK-NC_01\#e ns_id=1106055>.

Pierre Prier, "Israël n'a pas atteint tous ses objectifs à Gaza» Le Figaro (22 janvier 2009), en ligne: Le Figaro.fr <http:// www.lefigaro.fr/international/2009/01/23/01003-20090123ARTFIG000 04-israel-n-a-pas-atteint-tous-ses-objectifs-a-gaza-.php>.

Michel Bôle-Richard, "Opération "Plomb durci" : quelle "victoire" pour Israël?», Le Monde (19 janvier 2009), en ligne : Le Monde.fr <http:// www.lemonde.fr/cgi-bin/ACHATS/acheter.cgi?offre=ARCHIVES\&type_ite m=ART_ARCH_30J\&objet_id=1067009\&clef=ARC-TRK-NC_01\#ens_id=1 1 06055>.

François Béguin, "Les principaux objectifs d'Israël dans la bande de Gaza sont atteints", Le Monde (16 janvier 2009), en ligne: Le Monde.fr <http:// www.lemonde.fr/cgi-bin/ACHATS/acheter.cgi? offre=ARCHIVES \&type_item=ART_ARCH_30J\&objet_id=1066565\&clef=ARC-TRK-NC_01\#e ns_id=11 06055>.

Marek Halter, "Ce que le chef du Hamas veut obtenir d'Israël par la force», Le Figaro (29 décembre 2008), en ligne : Le Figaro.fr <http:// www.lefigaro.fr/debats/2008/12/29/01005-20081229ARTFIG00268-ceque-le-chef-du-hamas-veut-obtenir-d-israel-par-la-force-.php>.

A. J. "Nous n'avons pas intérêt à détruire le Hamas", Le Figaro (13 janvier 2009), en ligne : Le Figaro.fr <http:// www.lefigaro.fr/international/2009/01/13/01003-20090113ARTFIG00 314-nous-n-avons-pas-interet-a-detruire-le-hamas-.php>. 
hormis les forces gouvernementales, restent imprécis, inconnus et tributaires du rapport de force et des enjeux des relations internationales.

En revanche, malgré les défaillances de l'opération militaire d'Israël dans sa guerre au Liban en 2006, tel que souligné dans le rapport Winograd ${ }^{30}$, force est de rappeler que la zone de déploiement de la FINUL et des FAL au Liban-Sud ne souffre pas d'insécurité ou de danger au niveau de lancement de roquettes vers Israël. Bien au contraire, lorsqu'il y a eu des tirs de roquettes vers Israël du Liban-Sud lors du dernier conflit armé israélo-gazaouite en janvier 2009, Hezbollah s'est empressé de rassurer qu'il n'est pas responsable de ces tirs et qu'il compte respecter les dispositions de la résolution 1701. En effet, "le Hezbollah fait clairement savoir au gouvernement libanais, qui compte l'un de ses ministres, qu'il n'est pas impliqué par le lancement de roquettes sur le nord d'Israël à partir du Liban", a déclaré le ministre libanais de l'Information Tarek Mitri. ${ }^{31}$

- Dans une étude publiée récemment sur la guerre à Gaza $^{32}$, Anthony $\mathrm{H}$. Cordesman considère que sur le plan tactique Hamas a été bien touché dans son arsenal militaire et dans ses structures souterraines,

30. "Rapport sur la guerre du Liban : Olmert ne démissionnera pas" (30 avril 2007), en ligne: TF1.LCI.fr <http:// tf1.lci.fr/infos/monde/moyenorient/0,,3438479,00-rapport-sur-guerre-liban-olmert-demissionnera.html>.

31. "Israël réplique à un tir de roquettes venant du sud du Liban", Le Monde avec AFP et Reuters (8 janvier 2009), en ligne: Le Monde.fr <http:// www.lemonde.fr/cgi-bin/ACHATS/acheter.cgi?offre=ARCHIVES\&type_ite m=ART_ARCH_30J\&objet_id=1065544\&clef=ARC-TRK-NC_01>.

32. Anthony H. Cordesman, "The War in Gaza : Tactical Gains, Strategic Defeat?", Center for Strategic \& International Studies (9 janvier 2009), en ligne: CSIS.org <http://www.csis.org/component/option,com _csis_pubs/task,view/id,5188/type,1/>. 
sans qu'il y ait eu toutefois un vrai aboutissement mettant fin à ses opérations militaires à l'encontre d'Israël. Par ailleurs, sur le plan stratégique, les accords d'assistance militaire signés par les ÉtatsUnis d'Amérique, la France, l'Allemagne et la Grande-Bretagne avec Israël laissent présager une nouvelle configuration et un élargissement de la présence de l'OTAN dans le sud-est de la Méditerranée, la mer Rouge et le golfe d'Éden. Est-ce que cela constitue une avancée militaire stratégique, dont les résultats sont à attendre ultérieurement, ou bien s'agit-il d'un simple appui diplomatique, à venir, concernant le contrôle de contrebande d'armes venant à Hamas de l'Iran ou de la Syrie? De toute façon, il nous semble que sur le plan stratégique, la guerre est encore loin d'être perdue par Israël.

\section{Conclusion}

En parlant de quête de stabilité aujourd'hui au MoyenOrient au lieu d'instaurer une paix durable, d'une part, on approche la question par étapes et par processus et, d'autre part, on examine la situation avec pragmatisme.

En effet, les résolutions 1701 et 1860 ont en commun cette approche pragmatique et par processus, puisqu'elles appellent en premier lieu à une cessation des hostilités, ensuite à un cessez-lefeu durable suivi de l'entrée en vigueur - de nouveau - de la trêve (accord d'armistice en vertu de la résolution 62 du C.S du 16 novembre 1948), comme étant, dans leur ensemble, des étapes pour l'instauration d'une paix globale, juste et durable au MoyenOrient.

D'où, les efforts diplomatiques qui sont déployés présentement et à moyen terme concernent notamment ces étapes provisoires instaurant la stabilité, beaucoup plus que la paix. 
Cette dernière, devant être fondée sur les résolutions pertinentes du C.S. ${ }^{33}$, reste tributaire de la diplomatie extraonusienne et des rapports de force régionaux et internationaux.

La présence d'une force internationale de maintien de la paix au Liban constitue toutefois une chance pour le rétablissement de l'autorité du gouvernement libanais sur son territoire et la reconstruction de l'État libanais, après des années de démembrement, de guerre civile et de tutelle syrienne. Encore faut-il faire prévaloir le monopole de l'usage de la force par l'État ${ }^{34}$, et non pas son partage avec les milices libanaises et non libanaises, pour une meilleure stabilité politique et sécuritaire, sans oublier la cessation de la violation de la souveraineté libanaise à travers le vol de l'aviation militaire israélienne dans l'espace aérien libanais. Devant ces défis, la stabilité libanaise est en cours de construction, mais reste précaire au regard des enjeux des relations internationales, tant sur le plan régional qu'à l'échelle mondiale.

Quant à Gaza, outre le processus de réconciliation interpalestinien qui n'est pas encore en cours, il convient de noter que les exigences des parties au conflit, Israël et Hamas, sont encore sans répondant : la cessation des tirs de roquettes de Gaza vers le Sud d'Israël, la levée du blocus israélien sur Gaza, la fin de la contrebande d'armes vers Gaza et le règlement de la question des prisonniers palestiniens et du soldat israélien enlevé.

Ce n'est qu'en passant par ces étapes provisoires que l'on peut acheminer dans le processus de paix et avancer dans la négociation des accords internationaux visant la création d'un État palestinien (paix contre territoire-accords d'Oslo de 1993) et le retour des réfugiés palestiniens "chez eux". Encore faut-il que les parties au différend, gouvernement israélien et Autorité palestinienne, soient convaincus du résultat de ce processus de

33. Les résolutions pertinentes citées par la 1701 et la 1860 sont : 242 (1967) du 22 novembre 1967, 338 (1973) du 22 octobre 1973, 1397 (2002) du 12 mars 2002, 1515 (2003) du 19 novembre 2003 et 1850 du 15 décembre 2008.

34. Rapport du Secrétaire général, supra note 5 au par. 32. 
paix et que cela n'entre pas en conflit avec leur doctrine (fondamentalisme musulman) ou leur promesse électorale (la droite fondamentaliste israélienne). Dans ce cas, ce n'est plus un conflit entre droits internes et droit international qui aurait lieu, mais plutôt entre le règlement pacifique des différends et le noncontrôle d'un cycle de violences meurtrières. 